\title{
Convective inertia effects in wall-bounded thin film flows
}

\author{
Adras Z. Szeri • Victoria Snyder
}

Received: 19 February 2005 / Accepted: 19 December 2005 / Published online: 24 October 2006

(C) Springer Science+Business Media B.V. 2006

\begin{abstract}
We apply the boundary layer equations to inertial flow in wall bounded films that might be characterized as 'thin', say $\varepsilon \leq 0.1$ where $\varepsilon$ is the ratio of the characteristic lengths, yet to which the lubrication approximation of Reynolds no longer applies. Two particular flow geometries are investigated, nominally parallel plates and nominally inclined plates, both with and without spatially periodic perturbation of the stationary plate. A Galerkin-B spline formulation of the governing equations is employed, and we rely on parametric continuation to obtain solutions at higher values of the Reynolds number. In particular, we are able to demonstrate that the boundary layer equations yield accurate results for a wide range of Reynolds numbers when the aspect ratio is less than $1 / 10$. We also find that in both nominally parallel and nominally inclined geometries the sign of the inertial force correction is determined by the film contour in the neighborhood of the exit, this result might have implications in the design of MEMS devices.
\end{abstract}

Keywords Thin-film approximation . Boundary-layer equations · Fluid inertia . Channel flow $\cdot$ Fluid mechanics

\footnotetext{
A. Z. Szeri $(\varangle) \cdot$ V. Snyder

University of Delaware,

Newark, DE 19716-3140, USA

e-mail: szeri@me.udel.edu
}

\section{Introduction}

The equations that describe the motion of viscous fluids are difficult to solve, thus, whenever feasible, we search for ways to simplify them. Lubricant flow in bearings affords particularly easy simplification because two widely differing length scales characterize conventional lubricant films. Reynolds took advantage of this when he developed the 'lubrication approximation' and constructed what is now called the Reynolds theory of lubrication [1]. In the ensuing decades, the lubrication approximation has been widely used far outside the confines of lubrication, becoming one of the corner stones of fluid mechanics [e.g. 2-5]. Now that research focus is being increasingly directed to flow on the small scale and in narrow channels, a new look at the thin film approximation is justified [6, 7].

The Reynolds theory is a low Reynolds number, quasi two-dimensional theory, valid when the ratio of the characteristic lengths is vanishingly small. It breaks down where there is a sudden change in film thickness, as this violates the small slope condition locally, or when the Reynolds number increases significantly above zero. It is of interest then to know at just what aspect ratio and Reynolds number will this breakdown occur. The first of these breakdowns of Reynolds' assumptions suggests extension to three-dimensional Stokes flow, while the second indicates that extension to two-dimensional Navier-Stokes flow might be appropriate. 
Investigation into the effects of fluid inertia in thin films has a long and distinguished history. The first applications of the boundary layer equations to lubrication problems employed the method of averaged inertia [8-11]. The boundary layer equations for wall bounded thin film flows were solved recently by Pozzi and Tognaccini [12]. DiPrima and Stuart [13] computed inertial corrections to the linearized Navier-Stokes problem at small clearance ratios and at small values of the modified Reynolds number; the zero order approximation is identical to lubrication theory. Malvano et al. [14] investigated the effect of the ram pressure at inlet. San Andres and Szeri [15] worked out an accurate numerical solution of the exact equations and applied it to the flow between eccentric cylinders.

Our objective in this paper is to investigate the range of applicability of the boundary layer equations to narrow channel flows. In Section 2, we discuss the various limits provided by the prevailing flow conditions. We then study the quasi two-dimensional flow model that is applicable to thin-film flows at non-zero Reynolds number. In Section 3, we present a stream functionGalerkin formulation of the model derived in Section 2 and compare its predictions with finite element solutions of the full Navier-Stokes equations. In Section 4, we discuss typical solutions for flow between both nominally parallel and nominally inclined wavy boundaries.

\section{Analytical}

The equations of motion and continuity governing the flow of constant property Newtonian fluid can be written as

$$
\begin{aligned}
& \rho \frac{\mathrm{d} v_{i}}{\mathrm{~d} t}=\frac{\partial}{\partial x_{j}}\left(-p \delta_{i j}+2 \mu D_{i j}\right) \\
& \frac{\partial v_{i}}{\partial x_{i}}=0
\end{aligned}
$$

where $D_{i j}=\frac{1}{2}\left(v_{i, j}+v_{j, i}\right)$ is the symmetric part of the velocity gradient tensor. These equations will be normalized with characteristic length scales $L_{x z}$ and $L_{y}$ and characteristic velocity scales $U_{*}$ and $V_{*}=$ $\left(L_{y} / L_{x z}\right) U_{*}$

$$
\left\{x_{1}, x_{2}, x_{3}\right\}=L_{x z}\{X, \varepsilon Y, Z\}
$$

Here $\varepsilon=L_{y} / L_{x z}$ is the ratio of characteristic lengths. Time and pressure are non-dimensionalized according to

$t=\left(\frac{U_{*}}{L_{x z}}\right) \tau, \quad p=\frac{\rho U_{*}^{2}}{R e^{*}} P$

where

$R e=\frac{U_{*} L_{y}}{v}$, and $R e^{*}=\varepsilon R e$,

are the Reynolds number and the reduced Reynolds number, respectively. Note that this choice of normalization leaves the continuity equation formally invariant and retains the pressure term in the limit $R e * \rightarrow 0$. Recasting the Navier-Stokes equations and the equation of continuity in terms of normalized variables, one obtains

$-\varepsilon^{2}\left(\frac{\partial^{2} U}{\partial X^{2}}+\frac{\partial^{2} U}{\partial Z^{2}}\right)+R e^{*} \frac{\mathrm{d} U}{\mathrm{~d} \tau}=-\frac{\partial P}{\partial X}+\frac{\partial^{2} U}{\partial Y^{2}}$

$\varepsilon^{2}\left\{-\varepsilon^{2}\left(\frac{\partial^{2} V}{\partial X^{2}}+\frac{\partial^{2} V}{\partial Z^{2}}\right)-\frac{\partial^{2} V}{\partial Y^{2}}+R e^{*} \frac{\mathrm{d} V}{\mathrm{~d} \tau}\right\}=-\frac{\partial P}{\partial Y}$

$-\varepsilon^{2}\left(\frac{\partial^{2} W}{\partial X^{2}}+\frac{\partial^{2} W}{\partial Z^{2}}\right)+R e^{*} \frac{\mathrm{d} W}{\mathrm{~d} \tau}=-\frac{\partial P}{\partial Z}+\frac{\partial^{2} W}{\partial Y^{2}}$

$\frac{\partial U}{\partial X}+\frac{\partial V}{\partial Y}+\frac{\partial W}{\partial Z}=0$

The system represented by Eqs. (3) and (4) contain two parameters, the aspect ratio $\varepsilon$ and the reduced Reynolds number $R e^{*}$. We will now investigate the significance of two asymptotic cases provided by limiting values of these parameters.

Case (A): $\operatorname{Re}^{*}>\varepsilon^{2} \rightarrow 0$

Neglecting terms multiplied by $\varepsilon^{2}$ Eq. (3) yields

$R e^{*} \frac{\mathrm{d} U}{\mathrm{~d} \tau}=-\frac{\partial P}{\partial X}+\frac{\partial^{2} U}{\partial Y^{2}}$

$P=P(X, Z, \tau)$

$R e^{*} \frac{\mathrm{d} W}{\mathrm{~d} \tau}=-\frac{\partial P}{\partial Z}+\frac{\partial^{2} W}{\partial Y^{2}}$

The second of these equations, Eq. (5b), informs that the flow is a quasi two-dimensional Navier-Stokes flow: the pressure is invariant along $L_{y}$. Clearly, it is not possible to characterize this flow by a single equation in pressure. However, the pressure may be eliminated by differentiation with respect of $y$, leaving three equations to solve for the remaining three unknowns $U, V, W$. 
Equations (5a-c) are recognized as the 3-dimensional boundary layer equations [16].

It is only a second approximation, $R e^{*} t o 0$, that makes possible the derivation of a single pressure equation, the Reynolds equation of lubrication $[1,17,18]$

$\frac{\partial}{\partial x}\left(h^{3} \frac{\partial p}{\partial x}\right)+\frac{\partial}{\partial z}\left(h^{3} \frac{\partial p}{\partial z}\right)=6 \mu U_{0} \frac{\partial h}{\partial x}$

Here $h$ is the film thickness and $U_{0}$ is the effective velocity of the surfaces, which has values

$U_{0}= \begin{cases}U_{1}+U_{2}, & \text { relative rotation } \\ U_{1}-U_{2}, & \text { relative translation }\end{cases}$

Case $(B): \varepsilon^{2}>R e^{*} \rightarrow 0$

Neglecting terms multiplied by $R e^{*}$ in Eq. (5) leads to a three-dimensional Stokes flow

$\nabla^{2} \mathrm{~V}=-\nabla P$

It is, again, not possible to characterize the flow by a single equation in pressure and the full three-dimensional problem must be solved. However, in lubrication this limit is of interest only in the most unusual of circumstances.

\section{Numerical}

For infinite extent of the flow domain in the $z$-direction in Eq. (5), the flow becomes quasi one-dimensional [8]

$$
\begin{aligned}
& R e^{*}\left(U \frac{\partial U}{\partial X}+V \frac{\partial U}{\partial Y}\right)=-\frac{\partial P}{\partial X}+\frac{\partial^{2} U}{\partial Y^{2}} \\
& \frac{\partial U}{\partial X}+\frac{\partial V}{\partial Y}=0
\end{aligned}
$$

Much of the calculations will relate to flow between inclined, nominally flat planes (the 'plane slider'). In terms of the outlet film thickness, $h_{1}$, and the various non-dimensional quantities, the flow geometry is shown in Fig. 1. Here we put $h_{1}=h\left(x_{1}\right)=L_{y} H_{1}$ and $h_{2}=h\left(x_{2}\right)=L_{y} H_{2}$ for the film thickness at outlet and inlet, respectively, and defined the length scales by $L_{y}=\left(h_{1}+h_{2}\right) / 2$ and $L_{x z}=B=x_{2}-x_{1}$.

Definition of a stream function

$U=\frac{\partial \Psi}{\partial Y}, \quad V=-\frac{\partial \Psi}{\partial X}$

and elimination of $P$ by differentiation leads to a single equation in $\Psi(X, Y)$. At this stage, we also introduce the change of variables

$$
\begin{aligned}
& \xi=X-X_{1} \\
& \eta=Y / H(X), \quad H(X)=h(x) / L_{y}
\end{aligned}
$$

and transform Eq. (8) to

$$
\begin{gathered}
R e^{*}\left(H \frac{\partial \Psi}{\partial \eta} \frac{\partial^{3} \Psi}{\partial \eta^{2} \partial \xi}-2 \frac{\mathrm{d} H}{\mathrm{~d} X} \frac{\partial \Psi}{\partial \eta} \frac{\partial^{2} \Psi}{\partial \eta^{2}}-H \frac{\partial \Psi}{\partial \xi} \frac{\partial^{3} \Psi}{\partial \eta^{3}}\right) \\
-\frac{\partial^{4} \Psi}{\partial \eta^{4}}=0, \quad 0 \leq \xi, \eta \leq 1
\end{gathered}
$$

The boundary conditions on the solid boundaries are

$$
\begin{array}{lll}
\Psi=0, & \frac{\partial \Psi}{\partial \eta}=-H, & \text { at } \eta=0 \\
\Psi=Q^{*}, & \frac{\partial \Psi}{\partial \eta}=0, & \text { at } \eta=1
\end{array}
$$

where $Q^{*}$ represents the flow rate.

The boundary condition specified for the upper plate, Eq. (12), introduces a new unknown, $Q^{*}$, into the set of unknowns. To insure that the problem remains mathematically well posed we need to increase the number of independent equations by one. We accomplish this by constraining the average pressure at outlet (ambient pressure) to be the same as the average pressure at inlet (ambient pressure):

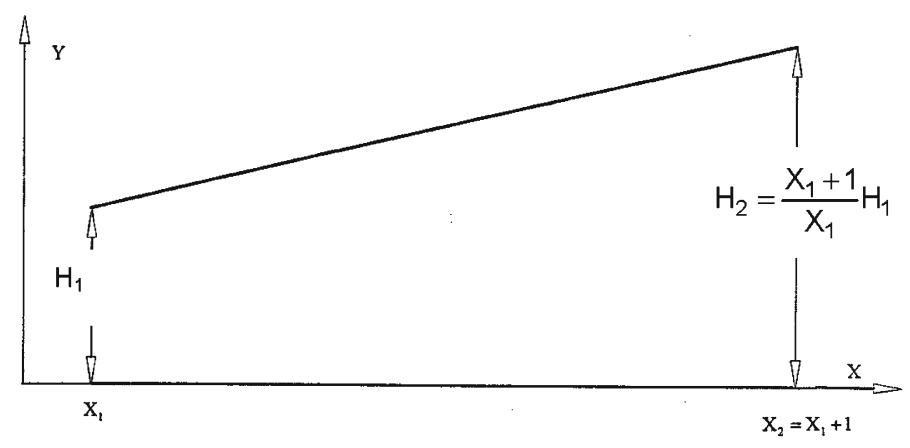

Fig. 1 Flow geometry 
$\int_{0}^{1} \int_{0}^{1} H(X) \frac{\partial P}{\partial \xi} \mathrm{d} \xi \mathrm{d} \eta=0$

We specify inflow and outflow boundary conditions at inlet, $X=X_{2}$, and outlet, $X=X_{1}$, respectively, by equating the second derivative of the $X$ component of the velocity, $U$, to zero at both outlet and inlet

$$
\left.\frac{\partial^{3} \Psi}{\partial X^{2} \partial Y}\right|_{\xi=0}=\left.\frac{\partial^{3} \Psi}{\partial X^{2} \partial Y}\right|_{\xi=1}=0
$$

We intend to approximate $\Psi(\xi, \eta)$, which is given only implicitly as solution of Eqs. (11-14), by piecewise polynomial functions [19] and apply Galerkin's method to evaluate the coefficients in the approximation. Denote the B-spline basis in $\xi$ by $A=\left\{A_{i}\right\}_{1}^{N_{\xi}}$ and in $\eta$ by $B=\left\{B_{i}\right\}_{1}^{N_{\eta}}$, then the basis for approximating $\Psi(\xi, \eta)$ is $A \otimes B$ and the expansion

$\Psi(\xi, \eta)=\sum_{i=1}^{N_{\xi}} \sum_{j=2}^{N_{\eta}} \psi_{i j} A_{i}(\xi) B_{j}(\eta)$

satisfies the equation of motion, Eq. (11), the pressure constraint, Eq. (13), and the boundary conditions, Eqs. (12) and (14), provided that the $\Psi_{i j}$ are chosen such that

(1) from equation of motion

$$
\begin{aligned}
& R e^{*} \sum_{i, m=1}^{N_{\xi}} \sum_{j, n=2}^{N_{\eta}} \psi_{i j} \psi_{m n}\left[\left(H_{r i m}^{(1)}-2 H_{r i m}^{(2)}\right) B_{s j n}^{(4)}\right. \\
& \left.+H_{r m i}^{(1)}\left(B_{j s n}^{(4)}+B_{s j n}^{(4)}\right)\right]+\sum_{i=1}^{N_{\xi}} \sum_{j=2}^{N_{\eta}} \psi_{i j} H_{r i}^{(3)} b_{s j}^{(5)}=0 \\
& 3 \leq r \leq N_{\xi}-2 ; \quad 3 \leq s \leq N_{\eta}-2
\end{aligned}
$$

(2) from pressure constraint

$$
\begin{aligned}
& R e^{*} \sum_{i, m=1}^{N_{\xi}} \sum_{j, n=2}^{N_{\eta}} \psi_{i j} \psi_{m n}\left[\left(h_{i m}^{(1)}-2 h_{i m}^{(2)}\right) b_{j n}^{(3)}-h_{m i}^{(1)} b_{j n}^{(2)}\right] \\
& \left.\sum_{i=1}^{N_{\xi}} \sum_{j=2}^{N_{\eta}} \psi_{i j} h_{i}^{(3)} B_{j}^{\prime \prime}\right|_{0} ^{1}=0 \\
& 1 \leq r \leq N_{\xi} ; \quad 2 \leq s \leq N_{\eta}
\end{aligned}
$$

(3) from conditions at solid boundaries:

$$
\begin{array}{ll}
\psi_{i 1}=0, & 1 \leq i \leq N_{\xi} \\
\sum_{i=1}^{N_{\xi}} \psi_{i 2} a_{m i}^{(0)}+c_{m}^{(1)}=0, & 2 \leq m \leq N_{\xi}-1 \\
\psi_{i N_{\eta}}=\psi_{i N_{\eta}-1}=Q *, & 1 \leq i \leq N
\end{array}
$$

(4) from entrance and exit conditions:

$$
\begin{aligned}
& \sum_{j=2}^{N_{\eta}} b_{n j}^{(0)} \sum_{i=1}^{N_{\xi}} \psi_{i j} A_{i}^{\prime \prime}(0)=0, \quad 3 \leq n \leq N_{\eta}-2 \\
& \sum_{j=2}^{N_{\eta}} b_{n j}^{(0)} \sum_{i=1}^{N_{\xi}} \psi_{i j} A_{i}^{\prime \prime}(1)=0, \quad 3 \leq n \leq N_{\eta}-2
\end{aligned}
$$

Here arrays such as $H_{\text {rim }}^{(1)}, \ldots, B_{s j n}^{(4)}, \ldots$ represent constant coefficients that result from an application of Galerkin's method to Eqs. (11-14).

The system of non-linear algebraic equations, Eqs. (16-20) can be written in the form

$G(\mu)=0, \quad \mu=(u, \sigma)$,

where $u$ is the vector of state variables $\left\{u_{i j k}, \Omega_{i j k}, w_{i j k}\right.$, $\left.p_{i j k}\right\}$ and $\sigma \in \sum$ is the vector of parameters $X_{1}, R e^{*}$, ... The computational scheme for solving Eq. (21), i.e., parametric continuation followed by the GaussNewton method, has been described elsewhere [15].

\section{Results and discussion}

The thin film approximation, one of the corner stones of fluid mechanics, has been used countless times and on a wide variety of problems. At vanishing reduced Reynolds number it yields the Reynolds theory of lubrication, while at large values Prandtl applied it to characterize the boundary layer next to a solid wall.

Lubrication problems are customarily modeled with the Reynolds equation, even when some of the assumptions of the underlying theory are invalidated through having a 'large' reduced Reynolds number $R e^{*}=\varepsilon R e$ or a 'large' aspect ratio $\varepsilon$, or even when the viscosity is strongly dependent on the pressure [20]. Many of these lubrication problems have great practical and economical significance; in spite of this, there are no theoretical or experimental guides as to the errors incurred when applying the Reynolds equation outside its domain, or what the first order extension of it should be when its assumptions are violated. Many researchers for example retain the zero modified Reynolds number assumption of the theory and look for removing the constraint on the aspect ratio. We, in contrast, intend to demonstrate that the zero Reynolds number assumption is easier to violate in lubrication practice than the assumption of thin film, and in such cases, the boundary layer equations, Eq. (5), could be applied. 
We wish to employ our stream function-Galerkin formulation of Eqs. (8) for the study of inertial flow in 'thin' films, say $\varepsilon \leq 0.1$. Before we can do that, however, two objectives must be met, validation of the model embodied in Eqs. (5), and validation of the numerical method employed for solving that model. To this end, we make use of both analytical and finite difference solutions of the Reynolds equation, Eq. (6), and finite element solutions of the complete NavierStokes and continuity equations, Eq. (1).

The principal conclusion from Eq. (5) is invariance of the pressure across the film. To investigate the upper bound of $\varepsilon$ for this conclusion to hold, we look at flow between inclined planes of various aspect ratios, as indicated in Fig. 1. As long as Eq. (5) holds, the pressure on the upper plate, $P(h)$, and the pressure on the lower plate, $P(0)$, is approximately equal, becoming identical in the limit $\varepsilon \rightarrow 0$. This idea may be made quantitative by introduction of a pressure difference coefficient, $d_{p}$

$d_{p}=|P(h)-P(0)|_{\max } / P(h)_{\max }$

where the subscript designates maximum values.

In Fig. 2, we indicate the value of $d_{p}$, calculated from the three-dimensional Navier-Stokes problem employing the finite element software FIDAP, as a function of the parameters $\varepsilon$ and $R e^{*}$. Attention here is arbitrarily restricted to $R e>10$, accepting this as a lower bound on the Reynolds number for applications. For 'small' values of the aspect ratio, Fig. 2 appears to support the assertion of Eq. (5): for $\varepsilon \leq 0.05, d_{p} \leq 0.01$, and even for the wider range $\varepsilon \leq 0.1, d_{p}<0.16$, though the increase in $d_{p}$ for $\varepsilon>0.05$ is quite rapid. Thus, for $\varepsilon \leq 0.1$, we have the approximate relationship $d_{p} \approx$ $\varphi\left(R e^{*}\right)$. This conclusion seems to hold well for $R e *$ $\leq 100$. Figure 3 plots the ratio of actual pressure maximum, $P_{\max }$, over its zero Reynolds number value, $P_{0, \text { max }}$, against $R e^{*}$, as calculated by FIDAP from the three- dimensional problem at various values of $\varepsilon \leq$ 0.1 . Data for different $\varepsilon$ values collapse onto a single curve, confirming, again, that under the stated conditions the aspect ratio is not a strong parameter of the flow, that is $P_{\max } / P_{0, \max } \approx \pi\left(R e^{*}\right)$.

Based on Figs. 2 and 3 we accept the boundary layer equations, Eqs. (5), as adequate for the characterization of thin film flows as long as $\varepsilon \leq 1 / 10$, though we will be returning to this question in Fig. 5 .

The next task is to provide evidence for the accuracy of the stream function-Galerkin solution of Eqs. (21). This will be attempted in Table 1 and Fig. 4 when $R e^{*}=0$, and in Fig. 5 when $R e *>0$.

Table 1 provides a comparison of non-dimensional forces, as calculated from our Galerkin formulation and from the Reynolds equation, respectively, for different channel geometries. Figure 4, on the other hand, contrasts pressure distributions for nominally parallel surfaces, one of which is perturbed sinusoidally, from the same two sources. The Reynolds equation is solved analytically in Table 1, and by the method of finite

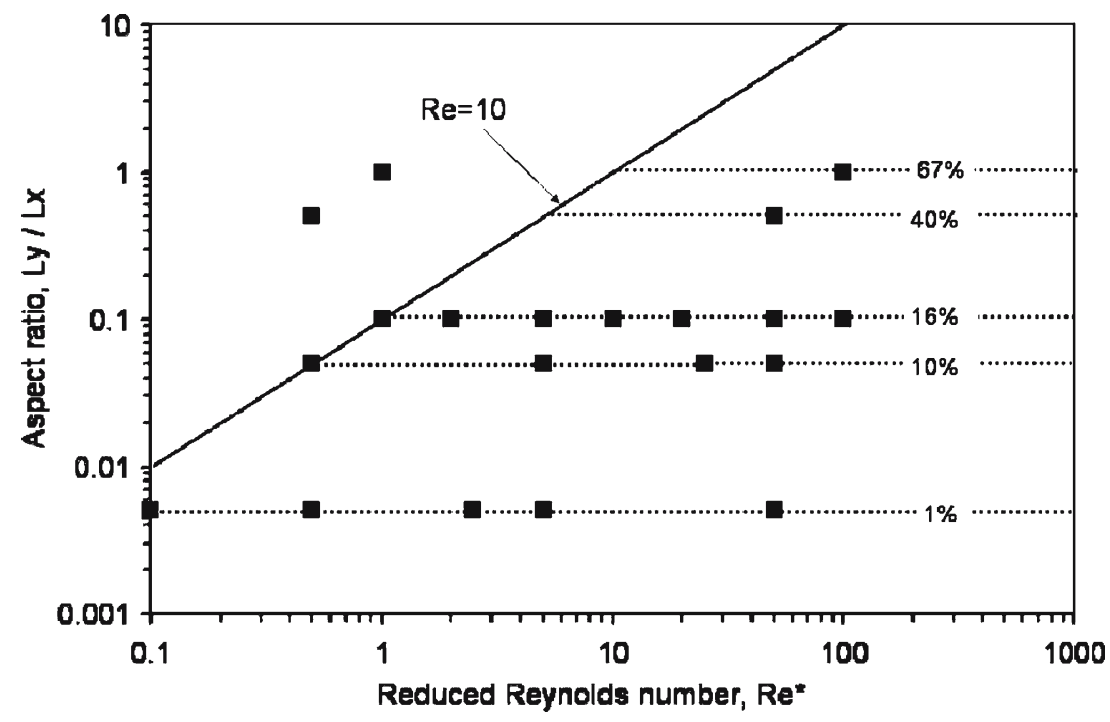

Fig. 2 Pressure difference coefficient $d_{p}$ for various values of the aspect ratio, $\varepsilon$, and reduced Reynolds number $R e^{*}$ (FIDAP) 


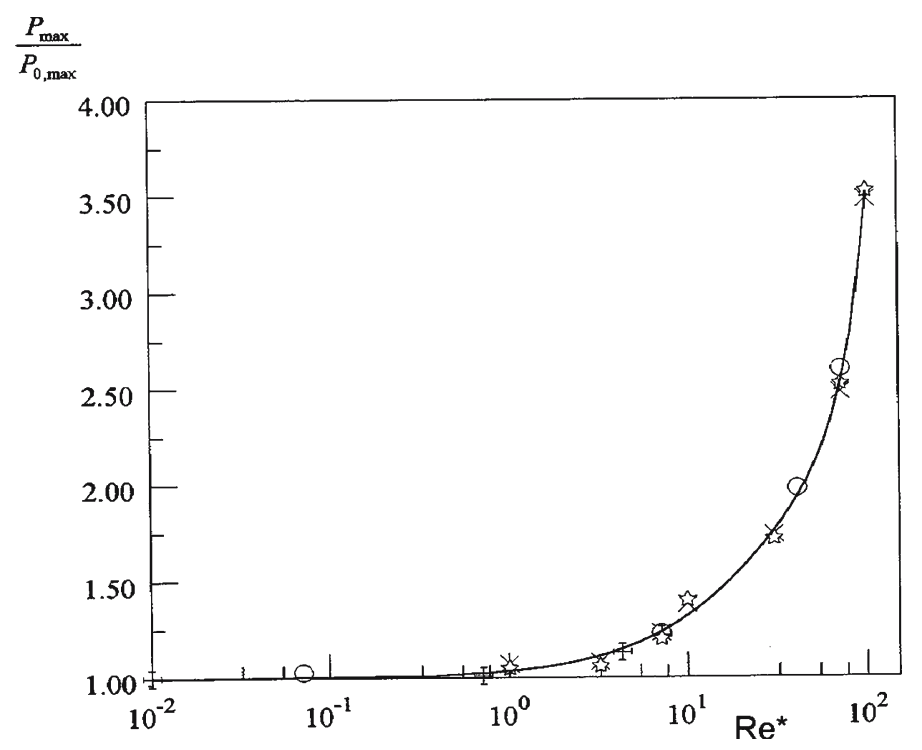

Fig. 3 Variation of $P_{\max } / P_{0, \max }$ with $R e^{*}, \operatorname{FIDAP}(+, \varepsilon=0.005 ; \mathrm{o}, \varepsilon=0.05 ; *, \varepsilon=0.08 ; \times, \varepsilon=0.1)$

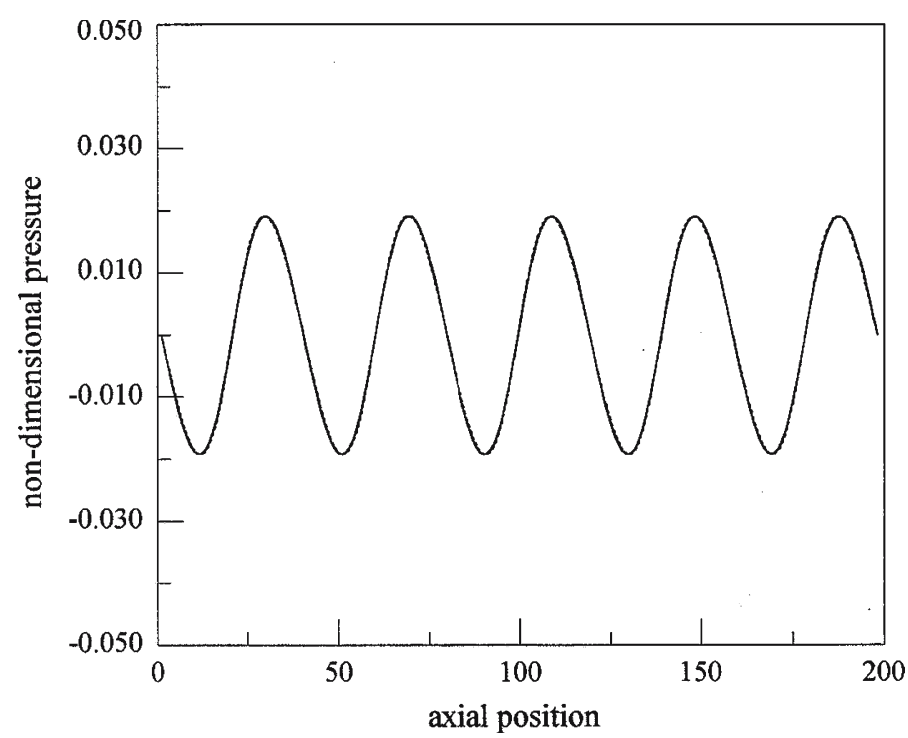

Fig. 4 Pressure distribution, nominally parallel plates $\left(\bar{X}_{1}=300, n=5, R e^{*}=0.0, \delta=0.1\right.$ :-, Galerkin's method; - - -, Reynolds equation)

differences in Fig. 4. In Fig. 5 we compare force $f$, at $R e^{*}>0$ from two sources, FIDAP solution of the full Navier-Stokes problem and our stream functionGalerkin formulation of Eq. (8). In this plot, we normalized the force with $f_{0}$, its value $R e^{*}=0$, and looked at two channel geometries, $h_{2} / h_{1}=2$ and $h_{2} / h_{1}=3 / 2$, under the condition $\varepsilon \leq 0.1$. It may be concluded from Table 1 and Figs. 4 and 5 that the stream function-Galerkin formulation is an acceptable method of solution of the problem.

Having demonstrated both the validity of the model, Eqs. (5), and the accuracy of our numerical method, Eq. (15), we proceed to investigate the influence of $R e^{*}$, that is the effect of convective inertia, on thin film flows. 


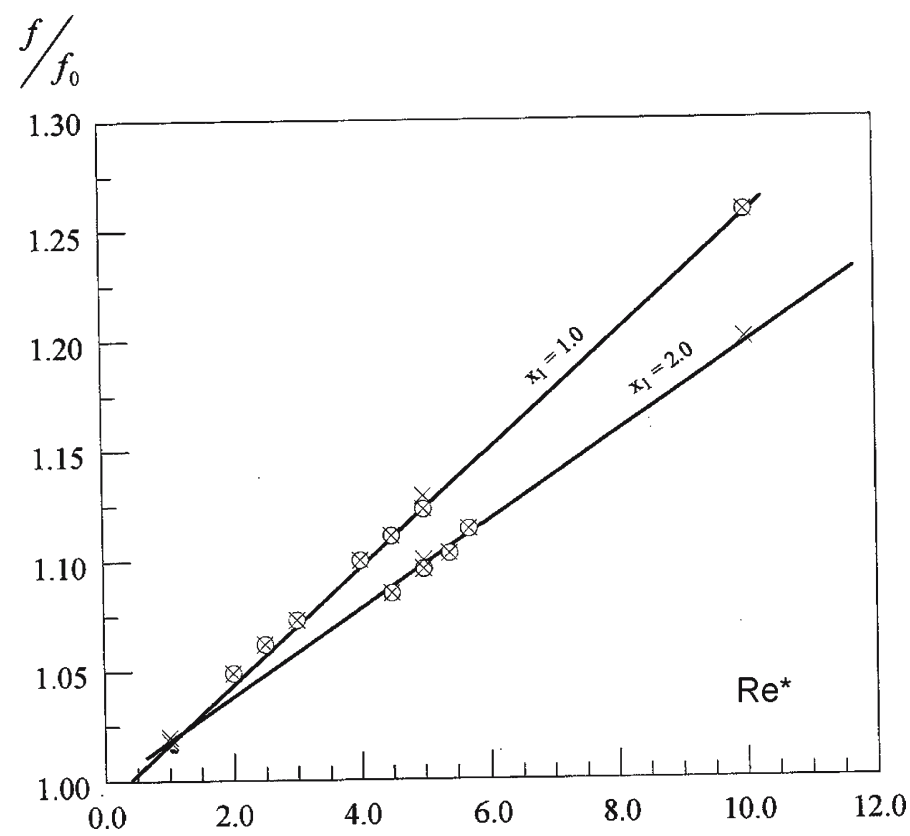

Fig. 5 Normalized force. Inclined flat plates, $\varepsilon \leq 0.1$. (o, Galerkins's method; $x$, FIDAP)

Table 1 Dimensionless force for plane slider $\left(R e^{*}=0\right)$

\begin{tabular}{rll}
\hline$h_{2} / h_{1}$ & Galerkin formulation & Reynolds Equation \\
\hline 11.00 & 1.3226 & 1.5795 \\
3.00 & 0.5658 & 0.5917 \\
2.25 & 0.4137 & 0.4228 \\
2.00 & 0.3521 & 0.3575 \\
1.50 & 0.2038 & 0.2049 \\
1.20 & 0.0913 & 0.0914 \\
1.10 & 0.0477 & 0.0477 \\
1.01 & 0.0050 & 0.0050 \\
\hline
\end{tabular}

\section{(a) Flow between nominally parallel planes}

We may note from Fig. 1 that parallel plate geometry can be approximated by $X_{1} \rightarrow \infty$. We take $X_{1}=300$, or $h_{2} / h_{1}=1.0033$, and perturb the film thickness according to $H(\xi)=X_{1}+\delta \cos (n \pi \xi)$. Figure 6 displays results for $n=5, \delta= \pm 0.1$ and $R e^{*}=4.0$. When $\delta>0$, the film shape is convergent (in the flow direction) at inlet and divergent at outlet, we characterize this as a c/d film shape. When $\delta<0$, the film shape is $\mathrm{d} / \mathrm{c}$. For $R e^{*}=0.0$ the average pressure is zero, but for film shape c/d the effect of convective fluid inertia is to lower the pressures below ambient within the channel, while for film shape $\mathrm{d} / \mathrm{c}$ the pressure is raised relative to inertialess flow. By changing to $n=5.5$, Fig. $7, \delta>0$ yields a d/d film shape, resulting in the channel walls being drawn together. In contrast, $\delta<0$ establishes a c/c film and a loading of the channel walls in the sense opposite to the $\mathrm{d} / \mathrm{d}$ film case, the walls are being forced apart. For diverging (converging) film shape at outlet, the effect of inertia is to decrease (increase) the pressure everywhere, thus creating a force that will tend to draw together (drive apart) the solid boundaries.

(b) Flow between nominally inclined planes

Setting $X_{1}=2.0$, we obtain the channel geometry $h_{2} / h_{1}=3 / 2$. Figure 8 displays results for $n=5$, $\delta= \pm 0.1$, and Fig. 9 for $n=5.5$ and $\delta= \pm 0.1$. The conclusions from these figures are similar to those drawn previously. The sign of the inertia force is dictated by the local film geometry approaching the outlet from upstream. Though the resulting forces are relatively small, in very narrow channels, such as occurring in MEMS, their effect may become significant and could be regulated by choosing the correct film profile at outlet. 


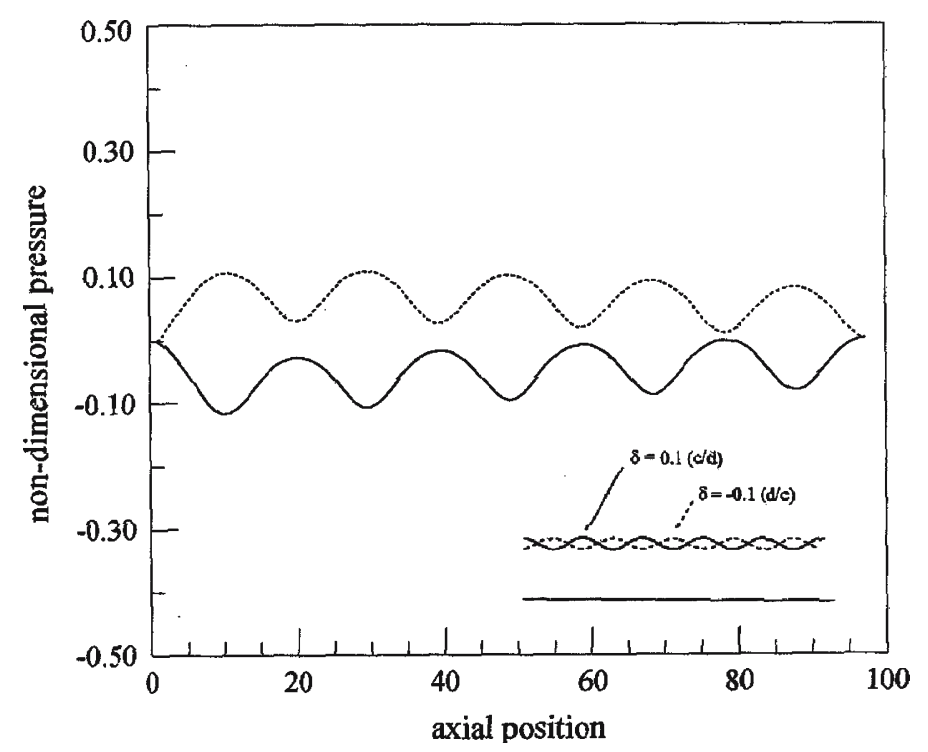

Fig. 6 Nominally parallel channel, Galerkin's method $\left(n=5, X_{1}=300, \delta= \pm 0.1, R e^{*}=4.0\right)$. Channel geometry:-convergent at inlet, divergent at outlet; $\cdots$ divergent at inlet, convergent at outlet



Fig. 7 Nominally parallel channel, Galerkin's method $\left(n=5.5, X_{1}=300, \delta= \pm 0.1, R e^{*}=4.0\right)$. Channel geometry:- divergent at, inlet divergent at outlet; $\cdots$ convergent at inlet, convergent at outlet 


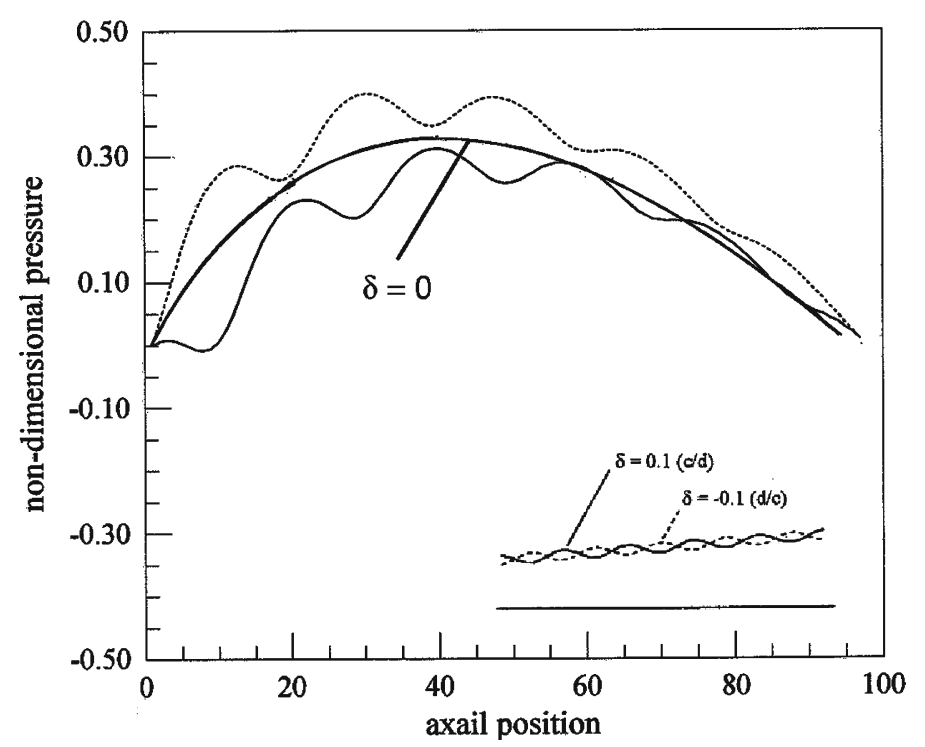

Fig. 8 Nominally inclined channel, Galerkin's method ( $\left.n=5, X_{1}=2.0, \delta= \pm 0.1, R e^{*}=4.0\right)$. Channel geometry:-convergent at inlet, divergent at outlet; $\cdots$ divergent at inlet, convergent at outlet

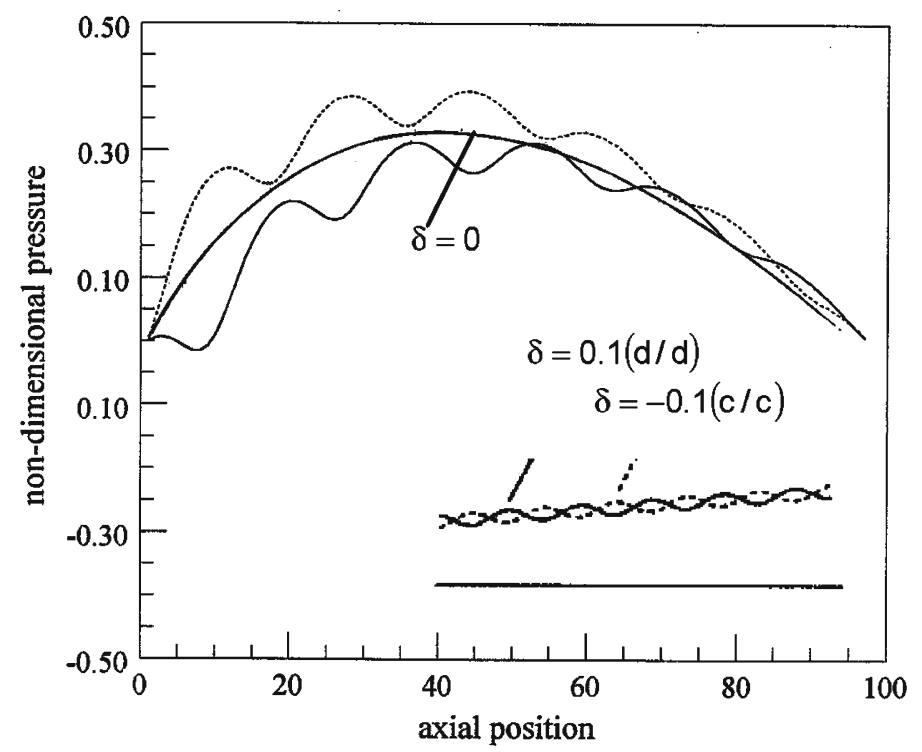

Fig. 9 Nominally inclined channel, Galerkin's method ( $\left.n=5.5, X_{1}=2.0, \delta= \pm 0.1, R e^{*}=4.0\right)$. Channel geometry:- divergent at inlet, divergent at outlet; $\cdots$ convergent at inlet convergent at outlet

\section{Conclusions}

We demonstrate here that the boundary layer equations, Eq. (5), may be applied with confidence to wallbounded thin film flows at non-zero Reynolds number, provided that the aspect ratio $\varepsilon$ does not exceed $1 / 10$. In that case, the normalized maximum pressure is a function of the reduced Reynolds number alone

$$
\frac{P_{\max }}{P_{0, \max }} \approx \pi\left(\mathrm{Re}^{*}\right) ; \varepsilon \leq 1 / 10
$$

We further find that in two-dimensional flow between nominally flat walls, one of which is periodically perturbed, the sign of the inertia force is dictated by local film shape approaching outlet, in the manner 
$\operatorname{sgn}\left(f-f_{0}\right)=-\left.\operatorname{sgn} \frac{\mathrm{d} h}{\mathrm{~d} x}\right|_{x_{\text {out }}} ;$

where $x$ is now increasing in the flow direction, i.e., $x>0$ when $U>0$.

\section{References}

1. Reynolds O (1886) On the theory of lubrication and its application to Mr. Tower's experiments. Philos Trans Roy Soc 177:157-234

2. Becker J, Grun G (2005) The thin film equation: recent advances and some new perspectives. J Phys Cond Matter 17:S291-S307

3. Schwartz L, Roy RV, Elly RR, Princen HM (2004) Surfactant-driven motion and splitting of droplets on a substrate. J Eng Math 50(2-3):157-175

4. Alvarez A, Soto R (2005) Dynamics of a suspension confined in a thin cell. Phys Fluids 17(9):Art. No. 093103

5. Munch A, Wagner B (2005) Contact-line instability of dewetting thin films. Physica D- Nonlin Phenomena 209(1-4): 178-190

6. Mishra C, Peles Y (2005) Flow visualization of cavitating flows through a rectangular slot micro-orifice ingrained in a micro-channel. Phys Fluids, 17(11):113602

7. Mukherjee S, Telekunta S, Mukherjee YX (2005) BEM modeling of damping forces on MEMS with thin plates. Eng Anal Boundary Elements 29(11):1000-1007

8. Osterle JF, Chou YT, Saibel E (1975) The effect of lubricant inertia in journal bearing lubrication. Trans ASME 79:494496
9. Constantinescu VN (1970) On the influence of inertia forces in turbulent and laminar self-acting films. J Lub Tech 92:473-481

10. Constantinescu VN, Galetuse S (1982) Operating characteristics of journal bearing in turbulent inertial flow. J Lub Tech 104:173-179

11. Launder BE, Leschziner M (1978) Flow in finite-width, thrust bearings including inertial effects, I and I. J Lub Tech 100:330-345

12. Pozzi A, Tognaccini R (2005) Influence of the Prandtl number on the unsteady thermo-fluid dynamic field around a thick plate. Meccanica 40:251-266

13. DiPrima RC, Stuart JT (1972) Non-local effects in the stability of flow between eccentric rotating cylinders. J Fluid Mech 54:393-415

14. Malvano R, Vatta F, Vigliani A (1999) Lubricated plane slider bearing: analytic and numerical approach. Meccanica 34:237-250

15. San Andres A, Szeri AZ (1985) Flow between eccentric rotating cylinders. J Appl Mech 51:869-878

16. Schlichting H (1968) Boundary layer theory, 4th edn. Mc-Graw-Hill

17. Szeri AZ (1998) Fluid film bearings: theory and design. Cambridge University Press

18. Dowson D (1998) Modeling of elastohydrodynamic lubrication of real solids by real liquids. Meccanica 33:47-57

19. de Boor C (1978) A practical guide to splines. Springer-Verlag

20. Rajagopal KR, Szeri AZ (2003) On an inconsistency in the derivation of the equations of elastohydrodynamic lubrication. Proc R Soc Lond A 459:2771-2786 